\title{
Supplementary Table: List of oligonucleotides used in this study
}

\begin{tabular}{|l|l|}
\hline \multicolumn{2}{|c|}{ List of oligos used to generate and confirm $\Delta$ kat $G$} \\
\hline F1 check & 5'-CTCGACATGTTCGGTGAGTG-3' \\
\hline R1 check & 5'-GTGAACAGCTCCTCGCCCTTCGACA-3' \\
\hline F2 check & 5'-CCTGCACGACTTCGAGGTGTTCGAG-3' \\
\hline R2 check & 5'-GAGAACGCCGAGATGAACG-3' \\
\hline F3 5' flanking & 5'-GATCACTAGTAGGCCGTCGAGGAATTCAAGAG-3' \\
\hline R3 5' flanking & 5'-GCTCATTTAAATCGATCGGGCGATCCTCAG-3' \\
\hline F4 3' flanking & 5'-GCTCTTAATTAAGCTTCGACGTCGCCTGACAC-3' \\
\hline R4 3' flanking & 5-'GACGATGCATGTGCAGCTGGTTGGACGTGTTG-3' \\
\hline \multicolumn{3}{|c|}{ Primers used to amplify and sequence Mtb katG } \\
\hline F5 KatG $M t b$ & 5'-ATCGAAGCTTGTTGGCGAGGCACCCTGTC-3' \\
\hline R5 KatG $M t b$ & 5'-ATCGGGTACCAAATCGCGCCGGGCAAACAC-3' \\
\hline F8 SEQ1 Kat G $M t b$ & 5'-TGTGGCCGGTCAAGAAGAAG-3' \\
\hline F9 SEQ2 Kat G $M t b$ & 5'-CTGCTGGCGCTTGGCAATAC-3' \\
\hline List of oligos used to generate katG and ndh variants in plasmid pMV761 and pCV125 respectively \\
\hline \multicolumn{2}{|c|}{} \\
\hline F6 KatG $M s m$ & 5'-ATCGGAATTCTCACAGCCCAATTCACCACTCC-3' \\
\hline R6 KatG $M s m$ & 5'-ATCGAAGCTTGGGCGTCCAGGTGGTTGTG-3' \\
\hline F7 ndh $m s m$ & 5'-ATCGAAGCTTGTTGATGATCGGCGGTAGCG-3' \\
\hline R7 ndh $m s m$ & 5'-ATGCGGTACCTTCGATGCGCCAGACCAAC-3' \\
\hline \multicolumn{2}{|c|}{ Primers used for site directed mutagenesis } \\
\hline F10 & 5'-GAACAGCTGGCCCGACTACGCGAGCCTGGACAAG-3' \\
\hline R10 & 5'-CTTGTCCAGGCTCGCGTAGTCGGGCCAGCTGTTC-3' \\
\hline
\end{tabular}

